\title{
Valoración médica de la sospecha de abuso sexual en personas menores de edad. A propósito del estudio de tres casos.
} Medical evaluation of sexual abuse suspicion in children. Report of three cases.

\author{
J. Gil Arrones', R. Ostos Serna², E. Largo Blanco3, L. Acosta Gordillo ${ }^{4}$ y \\ MA. Caballero Trigo ${ }^{5}$
}

\section{RESUMEN}

El diagnóstico, la valoración y el tratamiento de menores que pueden haber sido víctimas de alguna forma de abuso sexual, constituye un problema emergente de salud que tiene implicaciones legales y sociales. Por la complejidad y diversidad de los factores que intervienen, su abordaje tiene que ser llevado a cabo con la colaboración de diferentes especialistas, profesionales de distintas disciplinas y por instituciones de varios sectores.

A través de la revisión de tres casos se pretende expresar las dificultades que puede ofrecer el diagnóstico de abuso sexual en personas menores de edad, que como otros síndromes, se establece con un rango de probabilidad, basado en un proceso de valoración médica, psicológica y social; lo que no debe poner en cuestión el propio diagnóstico del abuso.

Como conclusión fundamental se extrae que no siempre es posible obtener indicadores de certeza, y que no siempre coincidirán la valoración médica con la psicológica o con la social, o que las actuaciones judiciales no habrán de ser en todos los casos determinantes para que la entidad pública pueda adoptar medidas de protección.

Palabras clave: Abuso infantil. Abuso sexual. Abordaje multidisciplinar.

Cuad Med Forense 2006; 12(43-44):57-74

\section{ABSTRACT}

The diagnostic, valuation and treatment of children that could have been victims of any form of sexual abuse, is an emergent health problem with important legal and social implications. Because of the complexity and diversity of the factors that can be related to this issue, its study has to be done with the cooperation of a wide range of specialists, professionals of different disciplines and also institutions belonging to several sectors.

Through the revision of three different cases our aim is to express the obstacles that can be created by the diagnostic of sexual abuse in minors. Such a diagnostic is established after a medical, psychological and social process, always in a probabilistic rank, still this fact must never create doubts about the diagnostic.

As a conclusion just to insist in the fact that not always it is going to be possible to obtain certainty indicators, and also that the medical impression will not always coincide with the psychological or social one, as well as not always it is necessary to have the permission of the Court to obtain a response of the Public Administration.

Key words: Child abuse. Sexual abuse. Multidisciplinary assessment.

Correspondencia: Dr. J. Gil Arrones. Programa de Pediatría Social. Hospital Universitario de Valme. Ctra. de Cádiz, s/n. 41014 Sevilla. juanm.gil.sspa@juntadeandalucia.es.

\footnotetext{
1 Pediatra, coordinador del Programa de Pediatría Social del Hospital de Valme.

2 Especialista en Obstetricia y Ginecóloga, médica adjunta.

3 MIR de segundo curso en Pediatría.

4 MIR de cuarto curso en Pediatría.

5 Trabajadora Social, responsable de la valoración social en la UTS del hospital.
} 


\section{INTRODUCCIÓN:}

UN PROBLEMA REAL CON UN MÉTODO DIAGNÓSTICO DIFERENTE.

El abuso sexual en menores de edad es un diagnóstico relativamente frecuente en la práctica clínica cuya prevalencia depende, en gran medida, del conocimiento y de la adecuada valoración que el profesional haga de los indicadores de sospecha $[1,2]$.

Desde nuestra experiencia, el diagnóstico inicial del abuso es siempre un diagnóstico de sospecha. La certeza o el grado de probabilidad se obtiene después de un proceso de valoración de los indicadores mediante el estudio médico, social y psicológico $[3,4,5]$. Es un diagnóstico que se sitúa en una escala de probabilidad, según la especificidad o confluencia de diferentes categorías de indicadores de sospecha [6]. Por desgracia pocos indicadores son concluyentes por sí mismos y puede llegar a ser muy difícil establecer el diagnóstico de abuso con certeza, con toda probabilidad $y$, desde luego, en muy pocas ocasiones es posible establecerlo en la primera entrevista o en la primera exploración sin asumir un excesivo margen de error. El diagnóstico se basa, pues, en una valoración de indicios no exenta de un factor de interpretación del profesional que, por otra parte, siempre ha sido reconocido y apreciado en el ejercicio de la medicina.

Dado que hemos de intervenir diferentes profesionales, es necesario compartir un concepto de abuso sexual a menores que permita dar respuestas a criterios legales y de salud. Un concepto basado en criterios psicológicos, médicos, sociales y legales $[7,8,9,10]$. Sería un error asociar el diagnóstico de abuso sexual de forma exclusiva a la existencia de contacto genital, a la presencia de lesiones o a la verbalización del menor o, incluso, hacer una correlación entre la gravedad de las lesiones y la gravedad del abuso. En cualquier caso, la gravedad del abuso estará en función del impacto orgánico, psicológico o social a corto o a largo plazo lo que, a su vez, estará en relación con las características individuales de la víctima $[6, \mathrm{l}$ I] .

Por otra parte, el abuso sexual es un diagnóstico de salud en sus aspectos orgánico, psicológico y social, como preconiza la OMS. Para reducir el margen de error en la valoración del posible abuso sexual en un menor, la valoración de su impacto y el tratamiento deben ser realizados con un planteamiento interdisciplinar y con la implicación de las instituciones con responsabilidad y competencias [12,13]. Ningún profesional puede abordar por sí mismo todos los componentes del diagnóstico o del tratamiento del abuso sexual a un menor sin tener que asumir posibles errores que recaerían en mayor perjuicio de la víctima $[4,6,14,15,16,17,18,19,20]$.

UNA FORMA DE ORGANIZAR LA RESPUESTA.

En el hospital de Valme la respuesta a este problema está organizada a través de una estructura de apoyo al conjunto de los profesionales que, por ley $[21,22,23,13]$, tienen que actuar en un primer nivel de detección, valoración y asistencia a la víctima y por un equipo de segundo nivel que coordina el proceso asistencial de cada menor, integra la información y emite informes a las instituciones que procedan.

El Programa de Pediatría Social (PPS) del Área Hospitalaria de Valme ha adaptado un concepto que de respuesta al problema de salud que constituye el abuso sexual de menores y, a la vez, que permita colaborar con instituciones de Justicia y de Protección, en tanto que el abuso puede constituir un delito y el menor puede estar en situación de desamparo.

\section{LOS CONCEPTOS.}

EI PPS ha elaborado y difundido entre los profesionales una definición adaptada, simplificada, casi esquemática, partiendo de autores, publicaciones e instituciones de suficiente evidencia científica y reconocido prestigio, adaptándola a las referencias del Código Civil y del Código Penal español [24,25,26], (Tabla I). 


\section{Concepto de abuso sexual infantil}

Actuaciones de contenido sexual realizadas

en la persona de un menor de edad (18 años)

con asimetría de poder

(edad, jerarquía, profesión, violencia..)

bajo coacción, agresión o engaño (seducción) con la condición de ocultarlo.

Programa de Pecitatria Sacial. Area de Vaime

Tabla 1. Concepto de abuso sexual. (Adaptado de varios autores).

Es importante que en esta fase inicial se desligue al profesional de algunos mitos que inducen a no detectar o a no notificar: creer que no hubo "intencionalidad" o que se trata de "una familia normal". El concepto de intencionalidad y de responsabilidad del presunto autor no corresponde ser valorado por ningún médico.

De esa definición de abuso sexual en menores se han extraído algunos criterios que pueden ser de ayuda para que cualquier médico o enfermero del hospital pueda discernir inicialmente sobre la sospecha de abuso sexual, en la mayoría de los casos (Tabla 2). No obstante, la valoración definitiva debe analizar más profundamente situaciones que pueden estar en los límites mismos de lo que debe ser considerado abuso de menores; por ejemplo, una menor que a los 16 años decide irse de casa y tener relaciones con un señor de 29 años: ¿Sería suficiente la asimetría de edad para decidir si existe un abuso de la menor? En cualquier caso sería necesario completar un estudio de la menor, de su desarrollo, de sus capacidades, de su posible emancipación, del ambiente cultural de su familia, etc., a lo largo de un proceso respetuoso que, a la vez que eficaz, reduzca al máximo las molestias a la menor y a su familia.

\section{Criterios para valorar la sospecha inicial}

Víctima menor de 18 años

Intención de obtener satisfacción sexual

Acto oculto, pacto de silencio

Asimetría de edad > 4 años entre la víctima y

el presunto agresor

No consentimiento de la víctima si es < 13 años

Violencia, seducción o engaño

Programa de Pediatria Social. Área de Valme

Tabla 2. Criterios para valorar la sospecha inicial de abuso sexual en menores 


\section{EL CONOCIMIENTO DE LOS INDICADORES Y LA NOTIFICACIÓN DE LA SIMPLE SOSPECHA.}

Los profesionales de primer nivel conocen los indicadores de sospecha que el PPS ha resumido de forma didáctica $[27,28,4]$ (Tabla 3). Ante la presencia de cualquiera de estos indicadores, el médico lo registra en la historia clínica e informa a la Unidad de Trabajo Social (UTS) mediante hoja de interconsulta. En ese momento inicial valora si existe riesgo vital para el menor y la capacidad de protección que pudiera tener si vuelve con su familia. Si el posible abuso sexual ha podido ser reciente, antes de 48-72 horas, y si han podido existir lesiones u otros indicadores físicos, se programa una exploración coordinada en la que intervengan los especialistas que procedan y el Médico Forense.

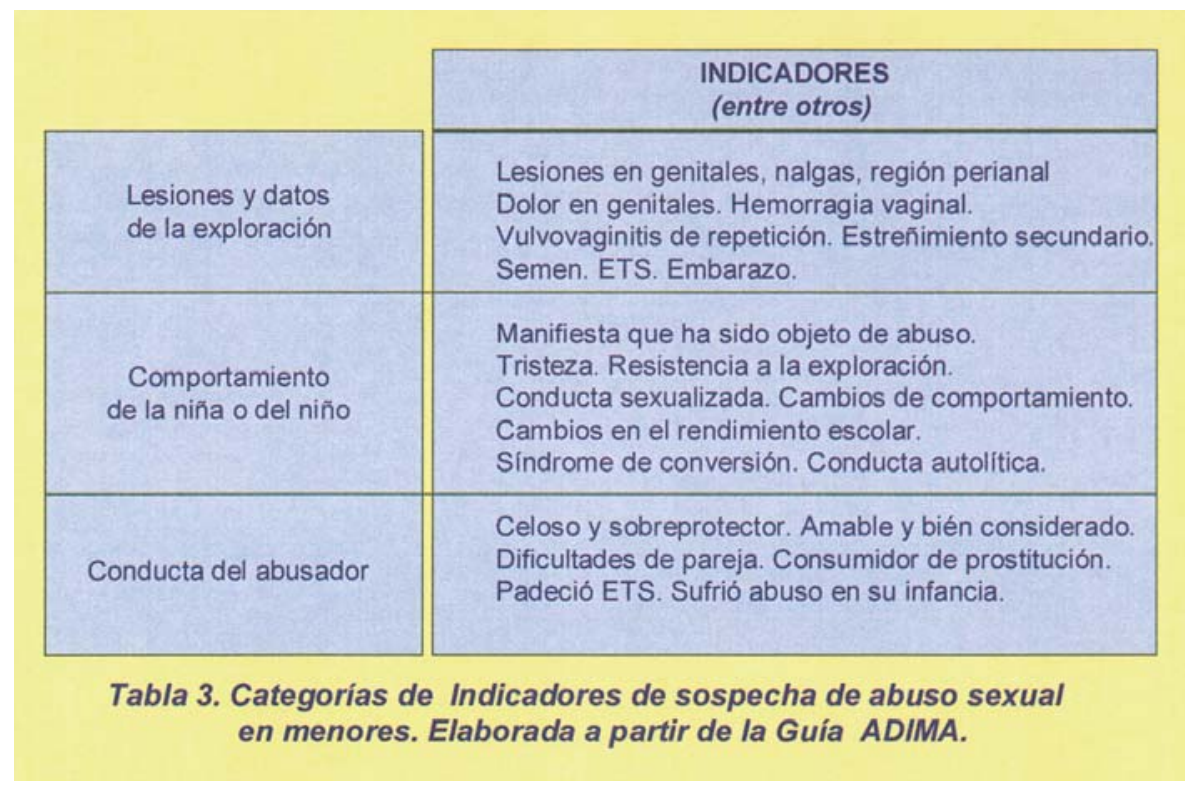

El diagnóstico inicial del abuso es siempre un diagnóstico de sospecha. Aunque existan indicadores físicos, lesiones aparentemente evidentes de abuso, o los indicadores sean muy "inespecíficos" de abuso. Aunque quepa la posibilidad de falsas alegaciones, el procedimiento más eficaz, en nuestra experiencia, consiste en recoger y notificar los indicadores de sospecha sin asumir inicialmente la confirmación del diagnóstico. La posibilidad de falsas alegaciones no debe ser argumento para no completar un estudio continuado.

Por otra parte, la ausencia de lesiones o de verbalización del abuso por parte del menor, la no coincidencia de las valoraciones médicas o psicológicas, no descarta la propia existencia de formas graves de abuso sexual que no dejó marcas, lesiones que ya desaparecieron o, formas de abuso que se produjeron sin contacto genital o físico.

\section{EL VALOR DE LOS INDICADORES.}

La valoración de los indicadores observados es una tarea compleja que debe ser desarrollada por varios profesionales a lo largo de un proceso. Ninguno de los indicadores, por sí mismo y fuera de contexto, puede descartar o confirmar la certeza del abuso. En la práctica empleamos una clasificación orientativa para la valoración de indicadores, que recuerda básicamente las tres categorías 
publicadas por Hymel y Jenny en 1996, que puede resultar útil siempre que se haya completado el estudio médico, social y psicológico del menor: indicadores compatibles, específicos y concluyentes de abuso sexual $[29,30,31,6]$ (Tabla 4).

\begin{tabular}{|c||l|l|}
\cline { 2 - 3 } Indicadores & \multicolumn{1}{|c|}{ Concepto } & \multicolumn{1}{c|}{ Ejemplos } \\
\hline COMPATIBLES & $\begin{array}{l}\text { Trastorno, enfermedades o lesiones que, } \\
\text { aunque pueden deberse a otras causas, } \\
\text { en determinadas circunstancias podrian ser } \\
\text { secundarios a alguna forma de abuso sexual. }\end{array}$ & $\begin{array}{l}\text { Trastornos del } \\
\text { comportamiento. } \\
\text { Fisuras perianales. } \\
\text { Hemorragia vaginal. }\end{array}$ \\
\hline ESPECIFICOS & $\begin{array}{l}\text { Trastorno,enfermedades o lesiones cuyo } \\
\text { mecanismo de producción más frecuente son } \\
\text { las prácticas abusivas; aunque pudieran ser } \\
\text { producidas por otra causa muy poco probable, } \\
\text { que no ha sido demostrada. }\end{array}$ & $\begin{array}{l}\text { Conducta sexualizada. } \\
\text { Relatos del menor no } \\
\text { confirmados. } \\
\text { ETS en niña oniño } \\
\text { de edad prepuberal, } \\
\text { descartada trasnmisión } \\
\text { vertical. }\end{array}$ \\
\hline CONCLUYENTES & $\begin{array}{l}\text { Trastorno, lesiones o enfermedades que sólo } \\
\text { pueden haber sido producidos por mecanismos } \\
\text { de abuso sexual. } \\
\text { Se han descartado accidentes y otras causas. }\end{array}$ & $\begin{array}{l}\text { Verbalización con } \\
\text { informe psicológico de } \\
\text { veracidad. } \\
\text { Rotura de hímen en } \\
\text { edad prepuberal. } \\
\text { De esfinter anal. }\end{array}$ \\
\hline
\end{tabular}

Tabla 4. Valoración de indicadores de abuso infantil. Gil Arrones J.

Por ejemplo: deben ser estudiadas todas las lesiones localizadas en genitales, en la cavidad bucal, en región perianal o en nalgas. Aunque no todas las lesiones deben ser consideradas producidas como consecuencia de una agresión sexual, deben incluirse en el diagnóstico diferencial y más, cuando se descartan o son poco probables otras enfermedades causales.

Por otra parte, las fisuras perianales pueden ser secundarias a estreñimiento, pero debe valorarse su evolución cuando se tratan adecuadamente y se normaliza la consistencia de las deposiciones. También las fisuras y lesiones producidas por la agresión sexual pueden producir estreñimiento secundario [32].

En este seguimiento que puede durar semanas o meses, el Pediatra debe estar especialmente atento a la aparición de otros indicadores que pudieran incrementar el valor de la sospecha.

Lo mismo diríamos de sangrados vaginales, vulvovaginitis de repetición, las molestias al andar o al defecar, las dilataciones del orificio himeneal, del esfínter anal. Es necesario estudiar si existen causas orgánicas, funcionales o infecciosas y valorar su evolución después del tratamiento adecuado. Además se explorará la presencia de indicadores en su comportamiento, cambios en el rendimiento escolar, etc. [6]. Si se descartan otras causas y continúan los síntomas, debe informarse a la Unidad de Trabajo Social para que inicie una valoración del entorno familiar del menor. En estos casos se trata de sospecha basada en indicadores compatibles con el abuso.

Otros indicadores físicos obtenidos de la exploración del menor pueden considerarse específicos de las formas de abuso sexual y tener un alto valor predictivo del abuso. Es el caso de algunas enfermedades de transmisión por contacto sexual, embarazos en adolescentes; algunas lesiones vulvo-vaginales, anales, verbalización por parte del menor o conducta sexualizada, por ejemplo. Es posible que sea discutida la "especificidad" de algunos indicadores, algunas enfermedades de transmisión sexual, por ejemplo condilomas acuminados perianales, porque, en teoría, cabría la posibilidad de otros mecanismos de contagio $[33,34]$. En este tipo de situaciones es importante 
poner de manifiesto cuál es el mecanismo de contagio más probable y valorar el conjunto de todos los indicadores de sospecha que presenta.

Ante la presencia de indicadores "específicos", el abuso sexual no debe ser descartado del diagnóstico diferencial hasta que no se demuestre inequívocamente otro mecanismo causal diferente.

En estos casos debe iniciarse valoración social, asegurarse de la capacidad de protección familiar y, en caso de duda, informar a los servicios de protección de menores y al Juez, mediante parte de lesiones o informe. Debe iniciarse valoración social de la familia por parte de la UTS y la exploración psicológica del menor por psicólogos especilalizados.

Si se detectan indicadores concluyentes se debe informar simultáneamente al Juzgado de Guardia y a la UTS y, si existen dudas sobre la capacidad de protección de la familia o que el presunto autor pertenezca al ámbito familiar, se facilitará su ingreso hospitalario como medida de protección y se informará al Servicio Povincial de Protección de Menores [35] (a través de la UTS) por si procede decretar situación de desamparo. Una vez completado el estudio médico, social y psicológico, se puede afirmar el diagnóstico de certeza de abuso sexual.

\section{PROBABILIDAD DEL ABUSO SEXUAL.}

El diagnóstico de abuso sexual en un menor es siempre un diagnóstico de probabilidad $[5,6, \mid 4,15]$ que se puede establecer en una escala, que a efectos operativos facilita la expresión del diagnóstico: se descarta el abuso, es probable, existe alta probabilidad y certeza de abuso. (Tabla 5).

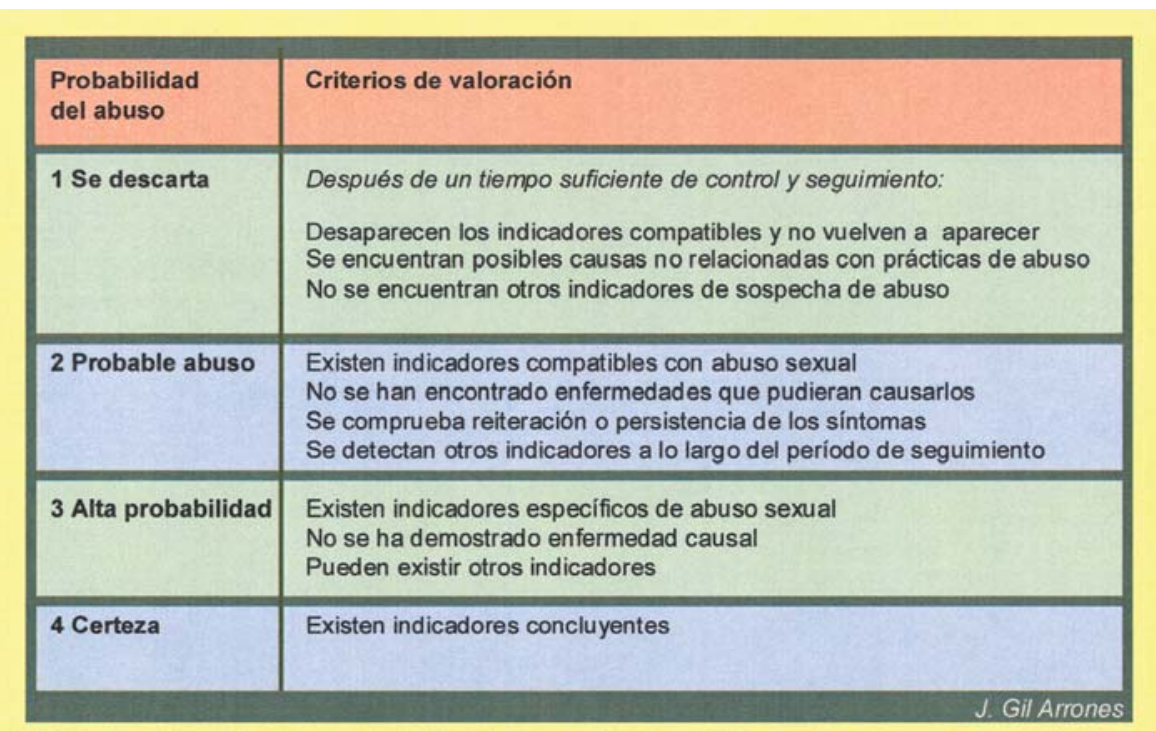

Tabla 5 . Escala de probabilidad diagnóstica del abuso sexual en menores

Es posible que no todas las instituciones y no todos los profesionales estén interesados en diagnósticos que dejen un margen de duda pero, desde el punto de vista de la salud y de la protección del menor, es importante iniciar un plan de trabajo que incluya seguimiento familiar, controles médicos, valoración del rendimiento escolar, de posibles cambios en su carácter, de posibles necesidades de tratamiento médico o psicológico, o la aparición de nuevos indicadores que incrementen la probabilidad del diagnóstico en términos objetivos. A veces la mejoría de su comportamiento 
cuando se inicia tratamiento psicológico o se adoptan medidas de protección, son suficientes para una confirmación clínica de la existencia del abuso.

\section{OBjetIVOS DEL ESTUDIO.}

A través del estudio de estos tres casos clínicos pretendemos:

I. Debatir sobre los límites en el concepto de abuso y de agresiones sexuales a menores.

2. Poner de manifiesto la complejidad de la valoración de indicadores y la necesidad de hacerlo dentro de un proceso continuado, integral y coordinado.

3. Reflexionar sobre las consecuencias de una valoración "restrictiva" de indicadores considerados como "poco específicos" de abuso sexual.

4. Presentar nuestra experiencia de trabajo interdisciplinar.

\section{MATERIAL Y MÉTODO:}

Estudio retrospectivo de tres casos clínicos extraídos del archivo del Programa de Pediatría Social del Hospital Universitario de Valme, estudiados por diferentes especialistas y profesionales.

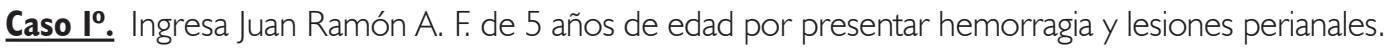
Ese mismo día, al llegar del colegio, le dice a su madre que "mientras estaba en el recreo, unos niños le empujaron, lo dejaron caer y se hizo daño en el culo", "estaban jugando y se cayó encima del balón". Mas tarde "le dolía" al defecar y "tenía sangre" al limpiarse. Los días previos su madre le notó cambios en su comportamiento: "no quería ir al colegio, ponía excusas, y que no quería ir, ...porque le pegaban". En otra ocasión le dice que "Daniel G., otro niño de su edad, le decía si jugaba o no jugaba". Si no jugaba se quedaba quieto, junto a la pared. Otro día le dijo que: "Felipe era quien decidía si jugaba o no". Daniel y Felipe eran compañeros del mismo aula.

El día siguiente a su ingreso, a las 24 horas de los hechos, Juan Ramón es explorado en presencia de sus padres: tiene crecimiento y desarrollo adecuados para su edad, previamente sano, no padece enfermedad crónica, no padece estreñimiento, ni toma medicamentos habitualmente. Tiene miedo a la simple inspección y a la toma de fotografías. Llora cuando sus padres se separan de él para la entrevista. Les dice que "no le va a contar nada"y que "no quiere hablar de eso". En la exploración por órganos y aparatos no presenta signos de padecer enfermedad. Genitales externos son normales para su edad y no presentan alteraciones. Se aprecian diferentes lesiones:

Ia: Equímosis redondeado de I $\mathrm{cm}$. de diámetro, de color parduzco, en tercio superior de cara externa de antebrazo izquierdo. 2a: Equímosis redondeado de $1 \mathrm{~cm}$. en tercio distal de cara anterior sobre radio derecho. $3^{\mathrm{a}}$ : Equímosis ovoideo de $3 \times 2 \mathrm{~cm}$. sobre cresta ilíaca izquierda. 4a: Da la impresión de dilatación de orificio analy de irregularidades en su contorno. $5^{a}$ : Herida lineal de $3 \mathrm{~cm}$. de orientación radial, a la una hora de la esfera, de bordes rojos. 6a: Herida lineal de $0,5 \mathrm{~cm}$. a las dos horas de la esfera. 7a. Herida lineal de 0,5 cm. a las tres horas. $8^{a}$ : Herida lineal, morfológicamente como una fisura que parte del contorno de orificio anal, a las cinco horas de la esfera. 9a: Otra herida lineal de unos $0,5 \mathrm{~cm}$. a las siete horas. $10^{\mathrm{a}}$ : Herida lineal de $1 \mathrm{~cm}$. en el borde izquierdo del contorno anal, a las nueve horas, en forma de escotadura profunda hacia el interior de orificio y de esfínter. I Ia: Herida erosiva con pérdida de epidermis, de forma triangular de $1 \times 2 \mathrm{~cm}$. con reacción inflamatoria a su alrededor, fuera del contorno anal, a las 5 horas de la esfera del reloj. (Figura I y 2 ). 


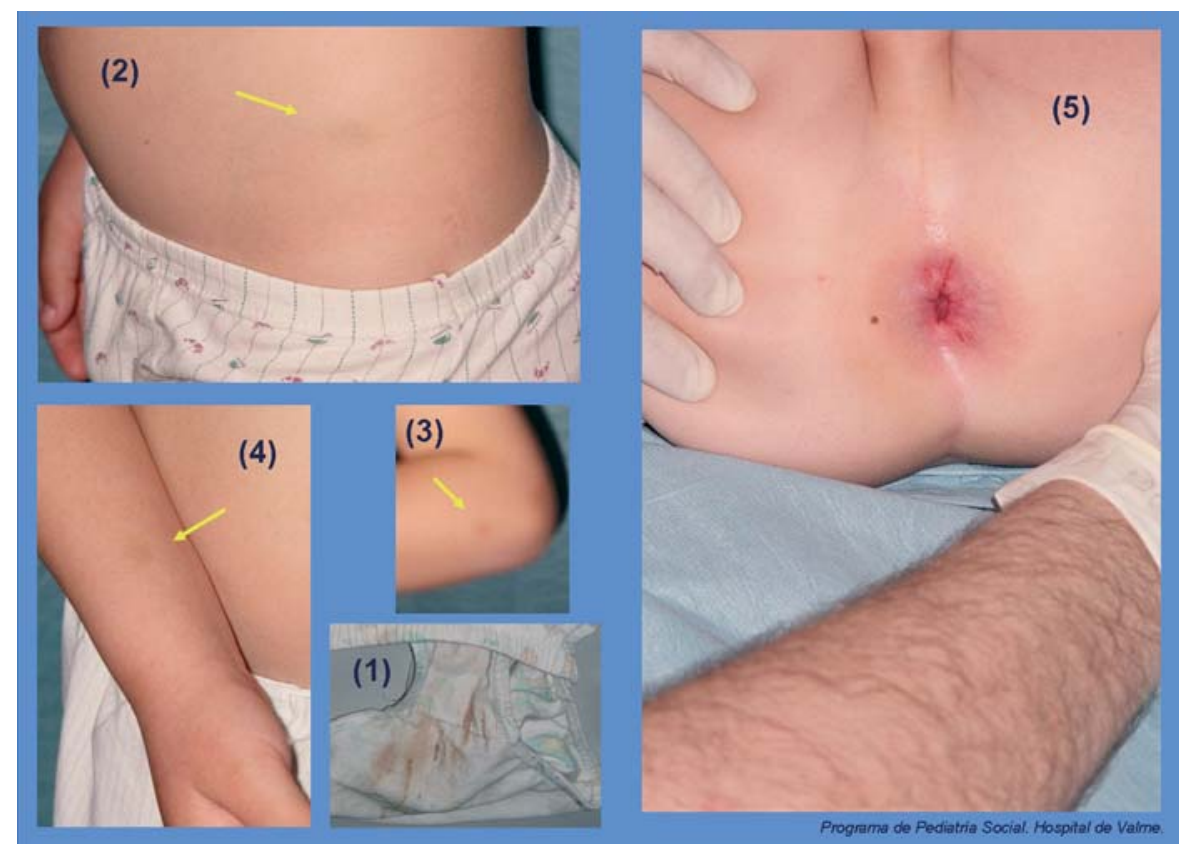

Figura 1. Caso 10: Niño de 5 años presenta sangrado anal que mancha la ropa interior (1), equímosis en cresta ilíaca izquierda (2), codo izquierdo (3) y en antebrazo derecho (4). Lesiones incisas y erosivas en región anal (5).

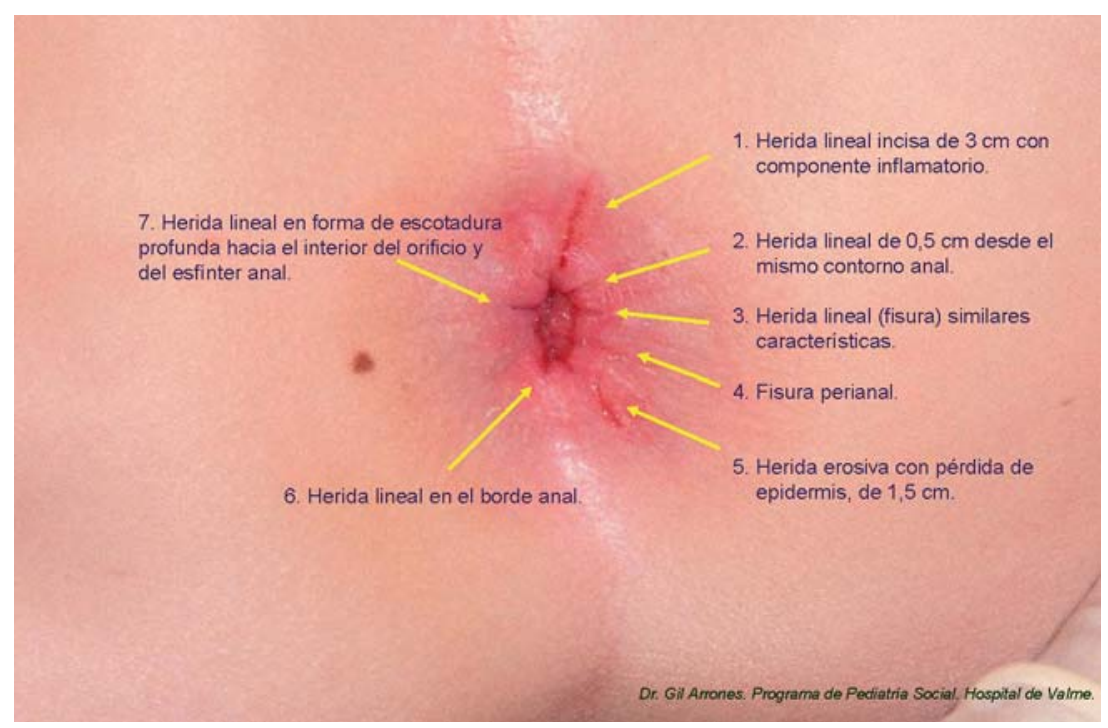

Figura 2. Caso 10: Detalle de las lesiones anales: diferentes heridas incisas, alguna erosiva, marcado componente inflamatorio y contuso en las lesiones superiores (entre las 10 y la 1 hora). 
Por las dificultades que el menor ofrece para la exploración, estas lesiones se aprecian mejor una vez que disponemos de las fotografías. A la vista de las mismas, se programa exploración endoscópica bajo sedación general, en presencia del Médico Forense, que se realiza tres días más tarde de esta primera exploración, cuando las lesiones tienen una data aproximada de cuatro días. Se precian cuatro lesiones con aspecto de desgarro en fase de cicatrización, sin signos de sangrado ni de lesiones internas al esfínter, que permanece aparentemente íntegro. Se observa: Ia: Herida lineal incisa de $3 \mathrm{~cm}$. de orientación radial, a la una hora de la esfera. 2a: Herida lineal que parte del contorno de orificio anal, a las cinco horas de la esfera. $3^{\text {a }}$. Herida lineal de unos $0,5 \mathrm{~cm}$. a las siete horas. 4a: Herida lineal incisa, de $1 \mathrm{~cm}$. en el borde izquierdo del contorno anal, a las nueve horas, más profunda hacia el orificio anal.

El resto de las lesiones han cicatrizado en el período transcurrido de 72 horas desde la primera exploración. (Figura 3). Durante su estancia en el hospital se le objetivaron molestias al sentarse.

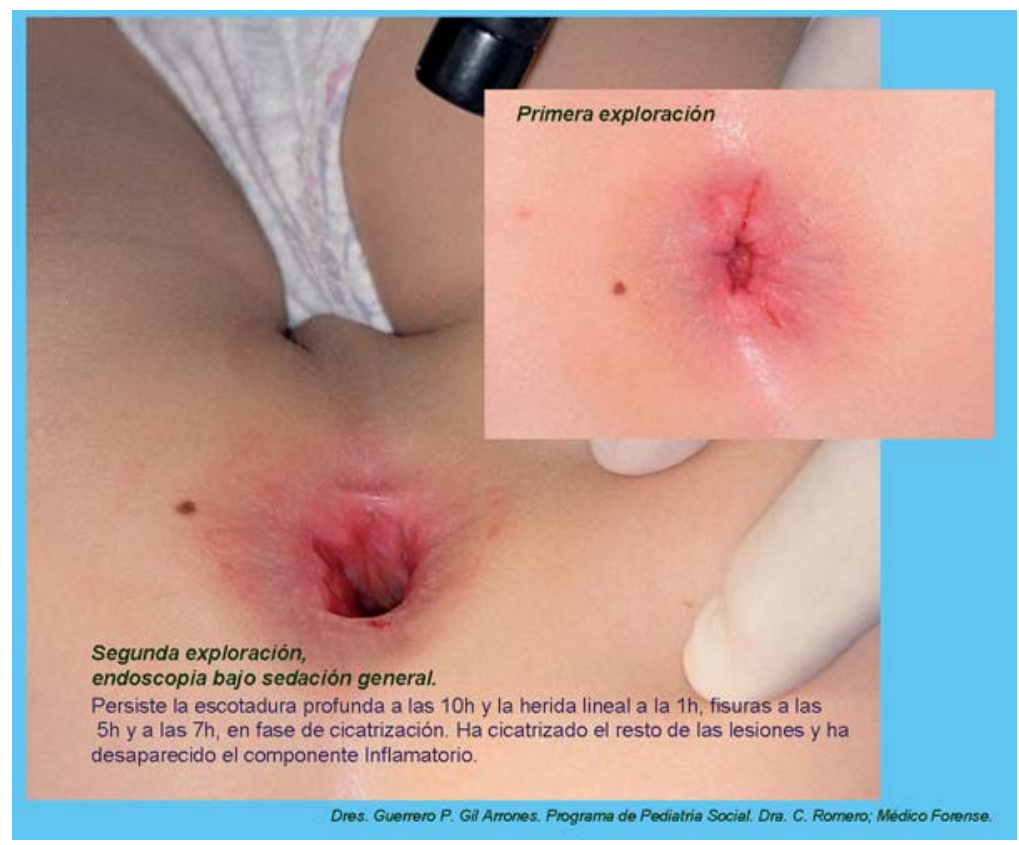

Figura 3. Caso 1: Evolución de las lesiones anales a los tres días de la primera exploración.

\section{Comentarios:}

Este menor presenta diferentes categorías de indicadores de sospecha de abuso sexual: cambios de conducta, lesiones y verbalización de la agresión.

- Indicadores físicos: sangrado y molestias al andar, lesiones en piel y en región perianal que no pueden haber sido producidas accidentalmente ni por ninguna enfermedad; con indicios de violencia con sujeción por terceras personas en antebrazos, codos y en cresta ilíaca. Son diferentes tipos de lesiones recientes, de una data similar. También ha presentado alteraciones funcionales derivadas de las lesiones: dolor y molestias al sentarse [32].

En un plazo de tres días han desaparecido siete de las lesiones que se observaron inicialmente, por lo que se deduce que las exploraciones médico-forense deben realizarse precozmente. 
- Trastornos de conducta que suponen un cambio en su trayectoria de comportamiento: tiene miedo a quedarse solo, a la exploración, a ir al colegio. Los cambios en su comportamiento constituyen otra categoría de indicadores de sospecha que responden a la vivencia traumática de una situación de estrés emocional (por acoso, exclusión, amenazas o agresiones) [12].

- Verbalización: En diferentes ocasiones ha manifestado a su madre que unos niños le empujaron mientras jugaba al balón, que "se hizo" daño en el culo; que le dolía al defecar. Ofrece datos sobre sus posibles agresores: Daniel y Felipe, dos de sus compañeros que decidían si jugaba o no. Estos hechos los sitúa en el colegio a la hora del recreo. También dice que no quiere hablar de "eso", que no le va a contar nada [16,35].

Las lesiones físicas son compatibles, en lo sustancial, con los relatos de la víctima: agresión producida probablemente en un acto único, en el entorno escolar, presuntamente por varios agresores también menores, bajándole la ropa interior que estaba manchada pero no rota.

\section{Valoración:}

Es un diagnóstico de certeza de agresión sexual en la persona de un menor, ocurrida probablemente en el ámbito escolar, presuntamente cometida por varias personas también menores, con un componente de exclusión o de acoso y probable falta de supervisión institucional.

No cabe duda que se trata de una agresión, entre iguales, con daño físico y emocional vivenciado por la víctima, que tiene un componente sexual por la localización de las lesiones, a pesar de las reservas en la valoración del carácter "sexual" que puede tener para niños de cinco años. Es difícil valorar, no es nuestra función, la responsabilidad de los niños agresores que, por otra parte, es evidente que tienen un problema de conducta que debe ser reconocido por sus padres y por las instituciones.

Caso $\mathbf{2}^{\circ}$. Niña de nueve años de edad, Inés R. G. ingresa para valoración de sangrado vaginal en varias ocasiones, durante varios meses de evolución. Se realiza valoración ginecológica por el equipo ginecológico de Urgencias apreciándole indicadores físicos de sospecha de abuso sexual: enrojecimiento del introito, secreción vaginal maloliente y lesiones escoriativas en ambas nalgas. La resistencia de la niña a la exploración, el miedo y la irritabilidad constituyen indicadores comportamentales que impiden completar la exploración, por lo que se programa exploración bajo anestesia.

En la exploración ginecológica realizada bajo sedación general en presencia del Médico Forense, se le aprecia enrojecimiento y maceración de la piel entorno a la vulva y al orificio anal, dos lesiones cicatrizadas, antiguas, en el borde del hímen que presenta un orificio dilatado y otras dos escotaduras en el borde himeneal a las 6 y a las 7 de la esfera horaria. También se obtiene material biológico (vello pubiano y secreciones) del interior de la vagina. Todos ellos constituyen signos inequívocos (certeza) de abuso sexual, dada la edad de la menor. (Figura 4).

Después de establecerse acogimiento familiar con familia extensa como medida de protección, exploración psicológica por EICAS y tratamiento psicológico por ADIMA, en uno de los controles médicos realizados sin previo aviso, diez meses más tarde, se le aprecian indicadores conductuales de sospecha (tristeza, miedo, rechazo a la exploración) que aconsejan nueva exploración ginecológica, para la que se obtiene la colaboración de la paciente y en la que se aprecian nuevas lesiones: hematoma superficial de aspecto contuso en ambas caras laterales del vestíbulo vaginal y la membrana himeneal es prácticamente inexistente. (Figura 5). 


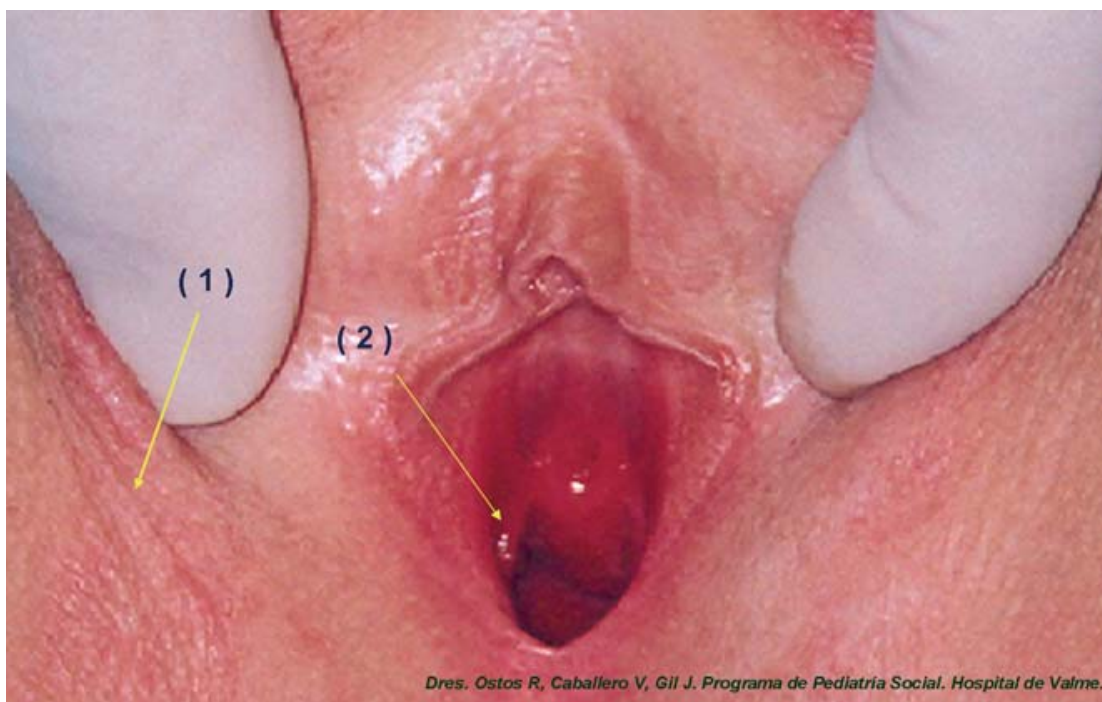

Figura 4. Caso $2^{\circ}$ : Niña de 9 años valorada por presentar sangrado vaginal. Por el rechazo a la exploración, esta se realizó bajo sedación general. Se aprecia enrojecimiento y maceración de piel entorno a vulva (1), orificio himeneal dilatado, lesiones cicatrizadas en el borde himeneal a las $6 \mathrm{~h}$ y a las $7 h$ y otras irregularidades (2). Del interior de la vagina se extrajo material orgánico: vello púbico y secreciones.

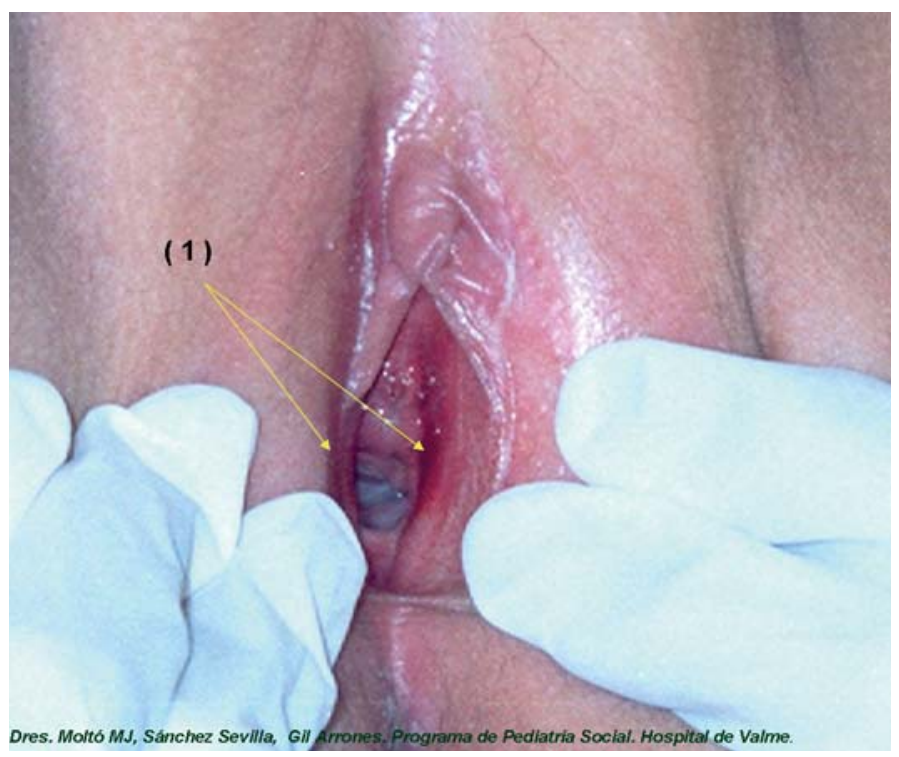

Figura 5. Caso 20: Segunda exploración ginecológica realizada en un control posterior por presentar tristeza, llanto fácil, miedo y rechazo a la exploración. Se aprecia hematoma superficial de aspecto contuso, reciente, en ambas caras de vestíbulo vaginal (1) y el hímen es prácticamente inexistente. 


\section{Comentarios:}

Esta niña ha presentado inicialmente una variedad de indicadores compatibles con la sospecha de abuso (hemorragia vaginal, tristeza, resistencia a la exploración, enrojecimiento, leucorrea, escoriaciones en nalgas) que han requerido una ampliación de las exploraciones en mejores condiciones (sedación general en quirófano); lo que ha permitido detectar indicadores altamente específicos, concluyentes, de abuso sexual (lesiones en el hímen, presencia de material biológico en vagina) y fundamentar el diagnóstico de certeza de abuso sexual $[37,38,1$ I ] .

El seguimiento médico (pediátrico y ginecológico) de esta menor, en colaboración con el equipo de tratamiento psicológico de ADIMA, ha sido fundamental para valorar la reiteración del abuso y la ineficacia de las medidas de protección adoptadas.

También se pone de manifiesto la imposibilidad de recuperar su salud sin garantizar su protección necesitando, incluso, la separación de su familia extensa.

\section{Valoración:}

Certeza de abuso sexual, basada en indicadores físicos concluyentes y en indicadores comportamentales específicos, reiterado, en situación de insuficiente protección en familia extensa. Se emite nuevo informe al Juzgado y al Servicio de Protección de Menores (SPM).

Caso $3^{\circ}$. Pedro M. R. fue derivado por su Pediatra cuando tenía 6 años y 10 meses de edad, para valoración por presentar encopresis, enuresis nocturna y 7-8 lesiones pequeñas compatibles con moluscum contagioso que presentaba únicamente en región perianal, que está enrojecida. También se le aprecia una verruga pediculada en tercio distal de brazo izquierdo. Dice su madre que el padre tiene "verrugas redondeadas como las del niño, aunque de mayor tamaño". Las deposiciones son de consistencia normal y algunas veces llega a controlar esfínteres. Ha estado en tratamiento psicológico en USMI durante 18 meses previos. Escolarizado con muy buenos resultados. Come mucho, presenta sobrepeso importante. El comportamiento en la consulta está por debajo de su edad, sobreprotegido, sin atender a normas disciplinarias. Un mes más tarde no han mejorado las lesiones a pesar de tratamiento tópico con Aldara (Imiquimod), aunque reconoce que no completa tratamiento por la irritación producida como efecto secundario. Meses más tarde persiste encopresis, usa pañales y presenta enuresis ocasionalmente.

Los trastornos de comportamiento continuaron siendo estudiado por la Unidad de Salud Mental Infantil (USMI) durante 5 años más, donde no ha verbalizado ni se han encontrado otros datos de sospecha de abuso. Ha sido estudiado en Consultas de Endocrinología y de Digestivo en el Hospital de Valme y del Hospital Infantil Virgen del Rocío donde se le repite manometría y técnicas de imagen que son normales y se le hace explícita (seis años más tarde) a la madre en presencia del niño, la posibilidad de abuso sexual como causa de los trastornos que presenta. Vuelve a presentar lesiones compatibles con moluscum contagioso que son biopsiadas y se obtiene diagnóstico histopatológico de condilomas acuminados. (Figura 6).

USMI vuelve a solicitar valoración de la probabilidad de abuso sexual, por el Programa de Pediatría Social, cuando tiene 12 años y 6 meses de edad. En una entrevista inicial en la que estaban presentes el niño, su hermana de 6 años, su madre y dos Trabajadoras Sociales, la madre me dice que Pedro le dijo: "si me ha hecho algo alguien, hace mucho tiempo, cuando era pequeño, pero no me acuerdo". En este contexto le pregunto directamente: i... pero tú sí te acuerdas? y me responde que sí. En otro momento posterior le pregunto si hace tiempo que le pasó, "lo del abuso", o ha sido reciente y responde que "hace tiempo". Pido autorización a su madre para derivar a estudio y tratamiento psicológico específico. 


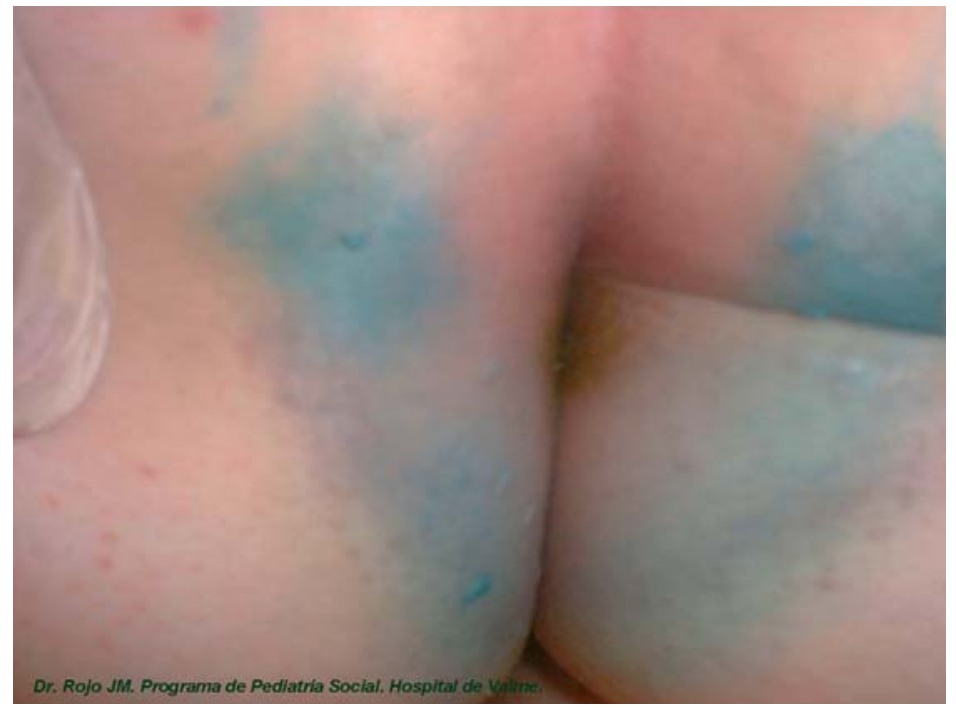

Figura 6. Caso $3^{0}$ : Lesiones condilomatosas perianales en niño de 12 años que ha presentado de forma recurrente, casi continua, desde los 6 años de edad con síntomas que podrían ser considerados somatomorfos: encopresis no orgánica y enuresis.

\section{Comentarios:}

Un universo de indicadores comportamentales, ambientales, físicos, funcionales, enfermedades de cuestionada transmisión por contacto sexual, el seguimiento por diferentes profesionales y especialistas no han sido suficientes para hacer el diagnóstico de abuso y prestarle la atención necesaria en un tiempo razonable.

A veces puede ser necesario un período excesivamente prolongado para descartar otras causas de los síntomas que ha presentado, para hacer un diagnóstico diferencial por exclusión, para el empleo de técnicas específicas de laboratorio, exploraciones psicológicas especializadas y para plantear el diagnóstico más probable de abuso.

En este caso, el diagnóstico de molouscum contagioso y de condilomas perianales no ha sido suficientemente valorado como enfermedad de transmisión sexual, por criterios basados en clasificaciones por el supuesto valor predictivo del abuso, de discutible rigor por lo que deberían ser revisadas $[39,40,30]$.

Por otra parte, la falta de verbalización del menor también ha sido excesivamente valorada a la hora de no establecer el diagnóstico de abuso.

\section{Valoración:}

Por la persistencia de los síntomas conductuales, funcionales, por el propio reconocimiento explícito del menor, a falta de ser confirmado por estudios psicológicos especializados, y por la continuidad de las lesiones condilomatosas, se establece un diagnóstico de alta probabilidad de abuso sexual, con contacto anal, reiterado, probablemente producido en el medio intrafamiliar.

No obstante, este menor podría desdecirse del reconocimiento del abuso que expresó en la consulta; lo que, si no se establecen medidas adecuadas de protección, sería más probable y afectaría a la credibilidad del diagnóstico por parte de otros profesionales, de otros sectores e instituciones. 


\section{RESULTADOS:}

Estos tres casos estudiados ponen de manifiesto que, mediante una estructura organizativa y funcional basada en un programa de apoyo a los profesionales, ha sido posible diagnosticar tres casos de abuso sexual que ofrecen importantes dificultades conceptuales y metodológicas. El Programa de Pediatría Social ha permitido que profesionales de diferentes Servicios asistenciales conozcan los indicadores de sospecha y los procedimientos para una actuación coordinada y realizadas con procedimientos adaptados.

Los procedimientos asistenciales se han adaptado a la necesaria inmediatez y coordinación de las exploraciones fuera de los cauces habituales para otros procesos, a las características de los menores, con acompañamiento de sus familiares, preservando el respeto a sus derechos y en colaboración con otras instituciones responsables.

En estos casos clínicos han sido reconocidos y valorados indicadores de diferentes categorías: conductuales, físicos (lesiones, infecciosos y alteraciones funcionales), los relatos del menor y la conducta de sus cuidadores o familiares; lo que ha sido fundamental para establecer el diagnóstico, iniciar el tratamiento y adoptar medidas de protección, según la capacidad familiar.

Algunos complejos diagnósticos de abuso y situaciones de desprotección han sido posibles por el trabajo coordinado y por el seguimiento de diferentes profesionales (Trabajadores Sociales, Psicólogos y Médicos de diferentes especialidades) del hospital y de otras instituciones.

Los controles médicos de seguimiento en colaboración con USMI, ADIMA y el SPM han permitido valorar mejor la evolución de salud de las víctimas y la eficacia de las medidas de protección.

\section{DISCUSIÓN:}

La exploración genital frecuentemente es normal o los hallazgos no son específicos en niñas y niños que han sufrido un abuso sexual, especialmente si el abuso no ha sido reciente [4 I], probablemente porque los abusadores se preocupan de no dejar marcas o porque las exploraciones se realizan cuando ya han curado las lesiones. Por ello es fundamental establecer protocolos de coordinación entre los Servicios de Urgencias y los especialistas en Pediatría y en Ginecología que detecten y valoren indicadores de sospecha y para que asuman su exploración y seguimiento.

El Programa de Pediatría Social es una estructura que permite coordinar las actuaciones y facilitar la rápida exploración, indispensable para el diagnóstico por el acelerado cambio que las lesiones experimentan en el tiempo. La exploración médica antes de 24-48 horas se recomienda en los niños y niñas tras un abuso sexual reciente, con lo que se incrementa la detección de lesiones físicas [42].

En algunas series solo el 4\% de los niños derivados para una evaluación médica por abuso sexual presentan lesiones físicas en el momento de la evaluación. Incluso, cuando se trata de un abuso en el que ha existido penetración vaginal o anal, la tasa de hallazgos físicos anormales según algunos autores puede ser solo del 5\% [3]. Los dos factores significativamente correlacionados en algunos estudios con la presencia de hallazgos anormales fueron el tiempo trascurrido desde el incidente y la historia de sangrado relacionado con molestias [43]. Por lo tanto, el diagnóstico del abuso sexual con contacto genital no solo debe fundamentarse en la observación de indicadores físicos en una única exploración.

En nuestra experiencia, que no siempre coincide con las series publicadas por otros autores, existe una elevada presencia de indicadores físicos; quizás porque las exploraciones se realizan en un breve plazo de tiempo, porque se consideran lesiones no permanentes y porque las exploraciones se producen simultáneamente por varios especialistas cada vez con mayor experiencia. 
Es fundamental una valoración global de las diferentes categorías de indicadores, tanto físicos como comportamentales y posible verbalización a lo largo de un proceso diagnóstico.

No todos los indicadores son concluyentes para el diagnóstico de abuso sexual, sin embargo la verbalización con informe psicológico de veracidad constituye un indicador de certeza tan fiable como la rotura del himen, o la presencia de una enfermedad de transmisión sexual en edad prepuberal, descartada la transmisión vertical en el momento del parto.

Con frecuencia se insiste en la verbalización del menor como "prueba evidente" del abuso, cuando los autores reconocen (Victoria Noguerol, Félix López) que solo una minoría de las víctimas verbalizan el abuso [16,2]. Algunos autores, como Finkelhor, afirman que la mayoría de las víctimas de abuso no presentan lesiones o marcas; no obstante, en nuestra experiencia tenemos un elevado porcentaje de casos en los que sí se detectan lesiones, algunas de ellas no permanentes, quizás porque examinamos precozmente a los menores con sospecha; pasadas 48 ó 72 horas desaparecen la mayoría de las evidencias físicas.

Algunas enfermedades como el moluscum contagioso no está contemplado como ETS y el diagnóstico diferencial con condilomas acuminados a veces es difícil y debe hacerse mediante examen histológico. A pesar de eso, el condiloma tampoco está reconocido en la bibliografía como un indicador de certeza, ni siquiera de alta probabilidad de abuso por lo que, con frecuencia, su presencia constituye un indicador de poco peso para establecer el diagnóstico de abuso [12,33,34].

La valoración restrictiva de algunos indicadores de sospecha catalogados a priori como poco predictivos del abuso (condilomas acuminados, por ejemplo) puede retrasar o dificultar el diagnóstico del abuso. En nuestra opinión y en nuestra experiencia de más de 250 casos estudiados, ante la presencia de estos indicadores deben emplearse argumentos suficientemente objetivos antes de proceder a descartarlos en el diagnóstico diferencial. Si se argumenta que (la lesión) pudiera haber sido producida por otra causa o por otro mecanismo, esta circunstancia debería ser probada.

Por ejemplo, en caso de la presencia de condilomas acuminados localizados únicamente en región perianal o en genitales de la víctima, que presenta de forma cronificada o recurrente y el posible autor o fuente de contagio tiene lesiones similares en genitales o en dedos de la mano, debería responder de forma convincente a algunas cuestiones y aportar pruebas: Si se produce por contacto de carácter no sexualizado, ¿Por qué el menor solo tiene lesiones en genitales o en zona anal y no en otras mucosas?

La enuresis y encopresis han sido valorados inicialmente como trastornos de comportamiento reactivos a la situación familiar con relaciones conflictivas, con escasa capacidad predictiva del abuso por ser indicadores poco específicos, máxime cuando existen factores ambientales y posibles enfermedades que pudieran justificar su aparición. No obstante, la tristeza es un síntoma que no suele faltar en las formas graves de maltrato y de abuso sexual.

El seguimiento psicológico, como el seguimiento médico, no siempre ha puesto de manifiesto indicadores de sospecha de abuso sexual, si nos referimos exclusivamente a la obtención de los relatos del menor sobre las sospechadas prácticas abusivas. De cara al diagnóstico y al pronóstico, podría ser de interés que el estudio psicológico aportara datos sobre la personalidad de la víctima y sobre su situación emocional, tal vez como consecuencia del impacto del abuso.

Parece evidente que existe mayor dificultad en el diagnóstico y valoración de los casos de abuso sexual ocurridos en el entorno familiar, intrafamiliares, a diferencia de los que son producidos por personas ajenas a la familia; al menos desde el punto de vista de los procedimientos y de los profesionales, aunque no para las víctimas. 
A través de estos casos se pone de manifiesto que el abuso o la agresión sexual entre iguales puede comenzar a edades muy tempranas, aunque pudiera ser discutible la intencionalidad y la supuesta responsabilidad que pudiera ser atribuida a los agresores y que, a pesar de su corta edad, desde la posición de la víctima puede calificarse el hecho de una verdadera agresión, con un componente sexual $[44,45,46]$.

\section{CONCLUSIONES:}

Primera. Generalmente no es posible confirmar o descartar el diagnóstico de abuso sexual a un menor en una única exploración, por un solo profesional. El diagnóstico de abuso sexual en un menor requiere un proceso de valoración médica, psicológica y social.

Segunda. La exploración física debe ser coordinada, adaptada a las características del menor y precoz, antes de las 48-72 horas siguientes al presunto abuso.

Tercera. La exploración psicológica debe ser realizada preferentemente por psicólogos o psiquiatras especializados.

Cuarta. La ausencia de indicadores físicos, de lesiones, o de verbalización del menor, no descarta la existencia de abuso sexual. El diagnóstico se basa en la valoración evolutiva del conjunto de indicadores. Cuando se aprecian lesiones o el menor relata prácticas abusivas, estos indicadores no deberían ser descartados sin argumentos bien fundamentados.

Quinta. Los indicadores de sospecha no siempre ofrecen un diagnóstico de certeza, sino de mayor o menor probabilidad que se verá incrementada por la presencia de varias categorías de indicadores o porque se descartan otras posibles causas en el diagnóstico diferencial.

A veces la evidencia del abuso se basa únicamente en la mala evolución de su salud emocional, de su comportamiento, de las lesiones o de enfermedades recurrentes, de la infelicidad en la que se instalan. A veces esperamos, exigimos, que los niños víctimas de abuso se comporten conforme a patrones que nosotros hemos previsto: que verbalicen el abuso, que lo mantengan a lo largo del tiempo ante jueces, médicos y psicólogos, que presenten lesiones inequívocas a pesar de que nuestras exploraciones se produzcan tarde y no tengan todo el rigor que debieran, a pesar de las instalaciones y de los procedimientos poco adaptados y de las limitaciones de nuestros sistemas; pero la realidad no suele ser así, ellos se comportan con respuestas adaptativas ante la agresión y ante la oferta social.

\section{AGRADECIMIENTOS:}

A todos los profesionales del hospital, de los centros de salud y de otras instituciones que han trabajado con nosotros en el estudio de niñas y niños con sospecha de haber sufrido abuso sexual:

Pediatras de los centros de salud del Área de Valme y de otras, a los médicos del Servicio de Urgencias, médicos y matronas del Servicio de Toco-ginecología, Enfermeras y Pediatras, Dermatólogos, Microbiólogos, Anestesistas, Digestólogos, Trabajadoras Sociales y de los S. Sociales Comunitarios, al SPM, a Jueces que han apostado por el bien superior del menor, a profesionales de instituciones como AMUVI, ADIMA, EICAS, VINCULOS, que han venido a cubrir un espacio fundamental en la defensa y en el tratamiento de las víctimas. A todas ellas por su trabajo con nosotros y porque, casi todo, lo hemos aprendido de ellos.

Y muy especialmente a las madres de niños y de niñas que han sido protectoras de sus hijos víctimas de abuso y que no han recibido la respuesta necesaria. 


\section{BIBLIOGRAFÍA:}

I. Finkelhor D: The international epidemiology of child sexual abuse. Child Abuse Negl, 1994;18:409-417.

2. López F, Carpintero E, Hernandez A, Martín MJ, Fuertes A: Prevalencia y consecuencia del abuso sexual al menor en España. Child Abuse?

3. Noguerol V: Aspectos piscológicos del abuso sexual infantil. En: Casado Flores ]: niños maltratados. Ed. Díaz de Santos, Madrid, 1997; 177-182.

4. Bays J, Chadwick D: The medical diagnosis of the sexually abused child. Child Abuse Neglect. 1993; 17: 91-110.

5. Caballero Trigo MA: Valoración inicial en los casos de abuso sexual infantil. Rev. Trabajo Social y Salud; Zaragoza, 1999: (33): 303-340.

6. Paradise JE: Valoración médica del niño que ha sufrido abuso sexual. Clin Ped de Nort Amer (ed. Esp.), 1990:889-912.

7. Gómez de Terreros I: Protección jurídica del menor. Los derchos del niño. En: Atención integral a la infancia con patología crónica. Ed. Alhulia. Granada, 2003: 69-88.

8. Noguerol V: Implicación del sistema legal en el abuso infantil. Un saco lleno de preguntas, dilemas y frustraciones. FAPMI 1995; l:95-104.

9. Guerra Arabolaza 0, Vaño Piedra C: Abusos sexuales: una situación dedesprotección. MEDIFAM 200I, II: 24-29.

10. López F: Abuso sexual: un problema desconocido. En: Casado Flores J: Niños maltratados. Ed. Díaz de Santos, Madrid 1997; |6|-167.

II. Vázquez Mezquita B: Agresión sexual. Evaluación y tratamiento en menores. Ed. Siglo XXI. Madrid, 1995; 20-24.

I2. ADIMA. Guía de atención al maltrato infantil. Sevilla, 1993.

13. Consejería de Asuntos Sociales. Procedimiento de coordinación para la atención a menores víctimas de malos tratos en Andalucía. Junta de Andalucía, 2003.

14. Américan Academy of Pediatrics: Committee of Child Abuse and Neglect: Guidelines for the evaluation of sexual abuse of children: subject review. Pediatrics 1999, 103, 2: 186-9I.

15. Atabaki S, Paradise JE. The medical evaluation of the sexually abused child: lessons from a decade of research. Pediatrics, 1999; (104) I78-185.

16. Noguerol V: Intervención ante el abuso sexual infantil. En: Oñorbe M, García Baquero M, Díaz Huertas J: Maltrato infantil. Prevención, diagnóstico y prevención desde el ámbito sanitario. Madrid, Consejería de Salud, 1995; 86-91.

17. Malacrea M: Trauma y reparación. El tratamiento del abuso sexual en la infancia. Barcelona: Paidós, 2000.

18. Cantón Duarte J, Cortés Arboleda MR: Guía para la evaluación del abuso sexual infantil. Ed. Pirámide S.A., Madrid, 2000.

19. De Paúl J, Arramburrena MI: Manual de protección infantil. Madrid: Ed. Masson, 1995.

20. Arramburrena MI, De Paúl J: Malos tratos a niños en la familia. Evaluación y tratamiento. Ed. Pirámide S.A., Madrid, 1997.
2I. Ley Orgánica I/1996 de Protección Jurídica del Menor.

22. Ley 14 /1986 General de Sanidad.

23. Consejería de Salud. Junta de Andalucía. Decreto 246/2005 por el que se regula el Derecho de las Personas Menores a recibir Atención Sanitaria Adaptada.

24. Bercowitz C: Abuso sexual en la infancia. Pediatrics in review, 1993,14(4):133-144.

25. Rubio B: Abuso sexual infantil. En: Díaz Huertas JA, Casado Flores J, Ruíz Díaz MA, Esteban Gómez J.: Atención al maltrato infantil desde el ámbito sanitario. Instituto Madrileño del Menor y la Familia. Madrid 1998: 109-II2.

26. Pou J, Jordan I, Gómez L: Abuso sexual infantil. Nuestra experiencia de un año. Anales Españoles de Pediatría, 1996:45(3): 245-248.

27. Finkelhor D: Epidemiological factors in the clinical identification of child sexual abuse. Child Abuse and Neglect, 1993:17:67-70.

28. Calvo Rosales J, Calvo Fernández J: Aspectos físicos del abuso sexual. En: Casado Flores ]: Niños maltratados. Ed. Díaz de Santos. Madrid 1997.169-175.

29. Hymel KP, Jenny C: Abuso sexual de niños. Pediatrics in review 1996;17(8):291-305.

30. García Algar 0, Mur Sierra A: Abuso sexual en la infancia: prevención de las enfermedades de transmisión sexual. Cuadernos de Pediatría Social, 2003, I:6-10.

31. Américan Academy of Pediatrics: Committee of Child Abuse and Neglect: "Páutas para la evaluación de niños sometidos a abusos sexuales. Pediatrics (edic española) 199I, 3I, 2: 85-90

32. Cann J, Vans ]: Perianal injuries resulting from sexual abuse: a longitudinal study. Pediatrics, 1993:91, 390-397.

33. Adams JA, Harper K: Examination fidings in legally confirmed child sexual abuse. I' ts normal to by normal. Pediatrics 1994; 94 (3): $310-317$

34. Américan Academy of Pediatrics: Sexually transmited diseases. En: Pickering LH, de 2000 Red Book: Report of the Committee on the infectious diseases. 25 de. Elk Grove Village, IL American Academy of Pediatrics. 2000: 143-147.

35. Decreto 3/2004, de 7 de enero: Sistema de Información Infantil de Andalucía. Consejería para la Igualdad y Bienestar Social. Junta de Andalucía.

36. Arruambarrena MI, De Paúl J: Maltrato a los niños en la familia. Evaluación y tratamiento. Ed. Pirámide. Madrid, 1994.

37. Wolf AS, Mittag JE: Ginecología pediátrica y juvenil. Atlas y guía para la consulta: abuso sexual en niñas. EDIMSA, 2000, 222-235.

38. Bannister A: From hearing to healing: working with the aftermath of child sexual abuse. UK: Longman, 1992.

39. Rojo JM, Gil J, Escudero J: Manifestaciones cutáneas del abuso sexual en la infancia. Piel, 2003,18, 2: 22-31. 
40. American Academy of Pediatrics: Sexually Transmited Diseases: Sexual Abuse. Report of the Commitee on infectious Diseases, 1997:II2-116

4I. Adams JA, Knudson S.: Genital findings in adolescent girls referred for suspected sexual abuse. Arch Pediatr Adolesc Med. 1996 Aug;:150 (8):850-7.

42. Palusci V], Cox E0, Shatz EM, Schultze JM: Urgent medical assessment after child sexual abuse. Child Abuse Negl. 2006 Apr;30(4):367-80.
43. Heger A, Ticson L, Velasquez 0, Bernier R.: Children referred for possible sexual abuse: medical findings in 2384 children. Child Abuse Negl. 2002 Jun;26 (6-7): 645-59.

44. AMUVI. Estudio de la violencia sexual. Sevilla, 2006.

45. Cirillo S, Blasio PD: Niños maltratados. Diagnóstico y terapia familiar. Ed. Paidós, Barcelona, 1997.

46. Save the Children Europe Group Project: research and reports relating to child sexual abuse. Daphne, 1997-2003. 